\title{
TANGENTIALLY AFFINE FOLIATIONS AND LEAFWISE AFFINE FUNCTIONS ON THE TORUS
}

\author{
In memory of Professor Itiro Tamura
}

BY TAKASHI INABA AND KAZUO MASUDA

\section{Introduction}

Let $\mathscr{I}$ be a foliation on a manifold $M$. We say that $\mathscr{F}$ is tangentiallyaffine if $M$ is covered by a collection of $\mathscr{I}$-distinguished charts for which the coordinate transformations are affine in the direction tangent to $\mathscr{F}$. This notion is, in a sense, dual to that of transversely affine foliation ([In]). Tangentially affine foliations appear in several branches of mathematics: for example, a Lagrangian foliation on a symplectic manifold is tangentially affine (See [AN]), and a supermanifold (in the sense of Rogers [Ro]) over a finite dimensional Grassmann algebra has a family of tangentially affine foliations ([BG], [RC1], [RC2], [CRT]).

The following problems naturally arise: (1) Which foliation admits a tangentially affine structure? (2) Given a tangentially affine foliation $\mathcal{I}$ on a compact manifold $M$, does there exist a leafwise affine function on $M$ which is nonconstant along leaves of $\mathscr{I}$ ? And if so, how many? Problem (1) is studied in $[\mathrm{Fu}]$ under additional condition that all leaves are affinely complete. As for (2), the authors cannot find any positive answer in the literature.

The purpose of this paper is to give complete answers to these problems for the 2-torus $T^{2}$. The results are as follows.

THEOREM 1. Every codimension one smooth foliation on $T^{2}$ admits a tangentially affine structure.

Example. There exists a tangentially affine foliation on $T^{2}$ admitting a leafwise affine function which is nonconstant along leaves.

THEOREM 2. Let $\mathscr{T}$ be a tangentially affine foliation on $T^{2}$ and $F$ a leafwise affine function on $T^{2}$ for $\mathcal{F}$. Then $F$ is uniquely determined by the values on the union of compact leaves of $\mathscr{T}$.

THEOREM 3. Let $\mathcal{T}$ be a tangentially affine foliation on $T^{2}$ and $L$ a compact

Received December 10, 1991. 
leaf of $\mathcal{I}$. Suppose that $L$ has a contracting holonomy on its right side. Let $U$ be a small right neighborhood of $L$ and $\mathcal{A}$ the vector space of all leaf wise affine functions on $U$. Then $\operatorname{dim} \mathcal{A}$ is 1,2 or $\infty$.

At the end of this paper we make a short remark on the 3-dimensional case :

PROPOSITION. The Reeb foliation on the 3-sphere admits no tangentially affine structure.

The authors would like to express their gratitude to A. Inoue for bringing the theory of supermanifolds to their attention. They would also be grateful to T. Mizutani, S. Matsumoto, N. Tsuchiya, A. Sato, Y. Mitsumatsu and H. Nakayama for helpful discussions.

\section{Definitions}

DEFINITION. Let $\mathscr{F}$ be a codimension $m$ foliation on an $(m+p)$-dimensional manifold $M . \mathscr{F}$ is tangentially affine if $M$ is covered by a collection of $\mathscr{F}$ distinguished charts with coordinates $(x, y) \in \boldsymbol{R}^{m} \times \boldsymbol{R}^{p}$ such that $\mathscr{F}$ is locally given by $x=$ constant and that the coordinate transformations are of the form $(\alpha(x), \beta(x, y))$, where $\beta$ is affine in $y$.

Observe that a tangentially affine foliation induces on each leaf an affine manifold structure.

DEFINITION. Let $\mathscr{F}$ be a tangentially affine foliation on a manifold $M$. A real valued function / on $M$ is leaf wise affine (resp. leaf wise polynomial, leaf wise constant) if, for each leaf $L$ of $\mathscr{T}$, the restriction of / to $L$ is affine (resp. polynomial, constant).

Remark. An affine manifold is a codimension 0 tangentially affine foliation. Polynomial functions on compact affine manifolds are studied in $[\mathrm{GH}]$ and $[\mathrm{Fr}]$.

Throughout this paper we denote by $\mathcal{C}$ the standard tangentially affine foliation on $\boldsymbol{R}^{2}$ by lines parallel to the $y$-axis.

\section{Proof of Theorem 1}

In this section we prove Theorem 1 . Let $\mathcal{F}$ be any smooth foliation of $T^{2}$. If $\mathscr{F}$ has no Reeb components, then $\mathscr{F}$ is the suspension of some diffeomorphism $g$ of $S^{1}(=\boldsymbol{R} / \boldsymbol{Z})$. Lift $g$ to a diffeomorphism $g$ of $R$ satisfying $\tilde{g}(x+1)=\tilde{g}(x)+1$, and define diffeomorphisms $T$ and $G$ of $\boldsymbol{R}^{2}$ by $T(x, y)=(x+l, y)$ and $G(x, y)=$ $(\tilde{g}(x), y+1)$ Then the quotient of $\left(\boldsymbol{R}^{2}, \mathcal{V}\right)$ by $T$ and $G$ is $\left(T^{2}, \mathscr{F}\right)$ with a tangentially affine structure induced from that of $\mathcal{C}$, as desired.

Next we consider the case where $\mathscr{I}$ has Reeb components. Let $L_{1}, \cdots, L_{r}$ be the boundary leaves of the Reeb components of $\mathscr{I}$ and $R_{1}, \cdots, R_{s}$ the com- 
pletions of the connected components of $T^{2}-L_{1} \cup \cdots \cup L_{r}$. Each $\left(R_{\imath}, \mathscr{F} \mid R_{i}\right)$ is either a Reeb component or a foliated $[0,1]$-bundle over $S^{1}$. If $R_{\imath}$ is a foliated $[0,1]$-bundle, denote the monodromy diffeomorphism by $g:[0,1] \rightarrow[0,1]$. By identifying the boundary points of $[0,1]^{2}$ by $G(x, y)=(g(x), y+1)$, we obtain from $\mathscr{V}$ a tangentially affine foliation on an annulus diffeomorphic to $\mathscr{T} R_{\imath}$. If $R_{2}$ is a Reeb component, let $g_{1}, g_{2}:[0, \varepsilon) \rightarrow[0, \varepsilon)$ be the holonomy contractions of the boundary leaves, where $\varepsilon>0$ is small. Let $A$ be the union of the half circle $(x-2)^{2}+y^{2}=4, x \geqq 2$, and the two line segments $y= \pm 2,0 \leqq x \leqq 2$. Let $(1 / 2) A=\{(x, y) \mid(2 x, 2 y) \in A\}$. Let $0<\delta \ll \varepsilon$ and choose smooth maps $h_{\imath}:(-\delta, \varepsilon+\delta)$ $\rightarrow \boldsymbol{R}(i=1,2)$ with the following properties: $h_{i}^{\prime}(s)>0$ for all $\mathrm{s}, h_{i}(s)<s$ for all $\mathrm{s}>0, h_{i}(s)=g_{i}(s)$ for $0 \leqq s<\delta$ and $h_{i}(s)=s / 2$ for $s>\varepsilon-\delta$. (Such maps clearly exist.) Let $T(x, y)=(x, y+2), H_{1}(x, y)=\left(h_{1}(x), y-1\right)$ and $H_{2}(x, y)=\left(h_{2}(x), y+1\right)$. We define a diffeomorphism $G$ from a small neighborhood of $A$ onto $(1 / 2) A$ as follows: $G(x, y)=T \circ H_{1} \circ T^{-1}(x, y)$ if $y>0$ and $x \leqq \varepsilon, G(x, y)=T^{-1} \circ H_{2} \circ T(x, y)$ if $y<0$ and $x \leqq \varepsilon$, and $G(x, y)=(x / 2, y / 2)$ if $x \geqq \varepsilon$. Denote by $B$ the region in $\boldsymbol{R}^{2}$ bounded by four curves $A,(1 / 2) A,\{0\} \times[1,2]$ and $\{0\} \times[-2,-1]$. On $B$, identify $A$ with $(1 / 2) A$ by $\mathrm{G}$. Then from $\mathcal{Q}$ we obtain a tangentially affine foliation on an annulus diffeomorphic to $\mathscr{F} \mid R_{\imath}$. Now for each $R_{\imath}, i=1, \cdots, \mathrm{s}$, choose a region $B_{\imath}$ and a diffeomorphism $G_{\imath}$ as above. By using translations and reflections, we can glue the corresponding sides of $B_{\imath}$ 's tangent to $C V$ and finally obtain a tangentially affine foliation on $T^{2}$ diffeomorphic to $\mathscr{T}$, as desired. This completes the proof.

Remark. A given foliation on $T^{2}$ may possess different tangentially affine structures (See the next section).

Remark. Unlike tangentially affine foliations, transversely affine foliations on $T^{2}$ must satisfy some strong topoiogical conditions (See [In] and [Ts]).

\section{Leafwise affine functions nonconstant along leaves}

In this section we construct two examples of tangentially affine foliations on $T^{2}$ which admit leafwise affine functions nonconstant on some leaves. In the first one, the foliation has no Reeb components and in the second one, the foliation consists of two Reeb components. Note that foliations constructed in the preceding section do not admit such leafwise affine functions, because all noncompact leaves of them are affinely unbounded. A somewhat "unusual" construction is needed to obtain the desired examples.

Example 1. Let $T$ be the diffeomorphism given by $T(x, y)=(x+2 \pi, y)$. Let $a(x)=x+1 / 2 \sin x$ and $B(x)=\cos \left(a^{-1}(x)\right)-\cos x$. For $x$ with $a(x) \neq x$, put $b(x)$ $=B(x) / B(a(x))$. Then it is easy to check that $b$ extends to a nowhere vanishing smooth function on $R$. Now we define a diffeomorphism $\mathrm{G}$ by $G(x, y)-$ $(a(x), b(x) y+1) . \quad G$ sends the $r$-axis to the line $\{y=1\}$. Identify the boundary 
points of $[-\pi, \pi] \times[0,1]$ by $T$ and $G$. Then we obtain from the product foliation $\mathscr{V}$ a tangentially affine foliation $\mathscr{F}$ on $T^{2}$. Observe that $\mathscr{F}$ contains two compact leaves. Define a leafwise affine function $F$ for $\mathscr{V}$ by $F(x, y)=B(x) y$ $+\cos x$. Then one can readily check that $F$ satisfies the equations $F=F \circ G$ and $F=F \circ T$. Hence $F$ induces a leafwise affine function $\bar{F}$ on $T^{2}$ for $\mathscr{T}$. Since $B$ vanishes exactly on the two compact leaves of $\mathscr{F}$, it follows that $\bar{F}$ is nonconstant along each noncompact leaf of $\mathscr{I}$, as desired.

Remark. Let $D=\boldsymbol{R} \times[0,1]$. The "developing image" $\cup_{n=-\infty}^{\infty} G^{n}(D)$ of $T^{2}$ is illustrated in Fig. 1. Observe that the affine structure of each noncompact leaf is a bounded interval, while the affine structure of each compact leaf is a quotient of a half line. Thus, in this foliation, a leaf of "bounded length" coils around a circle leaf endlessly!

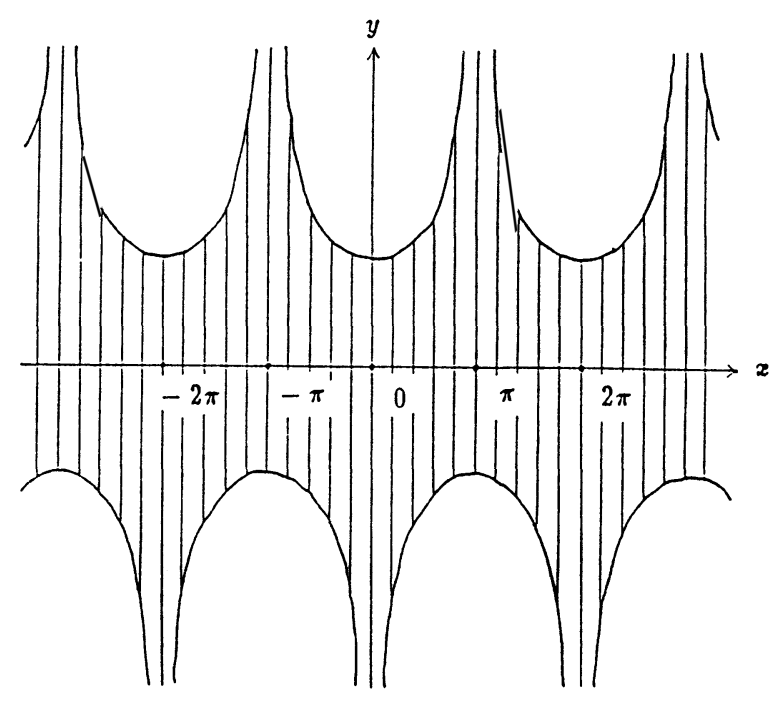

Fig. 1.

Example 2. Let $A$ be the union of two line segments $y- \pm 2, \mid x \leqq 2$, and two half circles $(x-2)^{2}+y^{2}=4, x \geqq 2$ and $(x+2)^{2}+y^{2}=4, x \leqq-2$. Let $(1 / 2) A=$ $\{(x, y) \mid(2 x, 2 y) \in A\}$. We will define $C^{\infty}$ even functions $b, c, B$ and $C$ so that they satisfy the following equations: (1) $c(x)+2 b(x)=1,(2) \quad B(x)=B(x / 2) b(x)$ and (3) $C(x)=B(x / 2) c(x)+C(x / 2)$ Let $0<\varepsilon \ll 1$ and let $\lambda: \boldsymbol{R} \rightarrow \boldsymbol{R}$ be a monotonic decreasing $C^{\infty}$ function such that $\lambda(x)=1$ for $x \leqq 1-(2 / 3) \varepsilon$ and $\lambda(x)=0$ for $|x| \geqq 1-\varepsilon / 3$. Set $C(x)=\lambda(x)\left(1-x^{2}\right)$. For $|x| \leqq 1-\varepsilon$, set $B(x)=3 x^{2} / 7$. Then by (3), $c(x)$ is uniquely determined for $0<|x| \leq 2-2 \varepsilon$ and $c(x)=-7$ if $0<|x| \leqq 1-\varepsilon$. So we define $c(0)=-7$. Now by $(1), b(x)$ is determined for $x \mid \leqq 2-2 \varepsilon$, hence, by (2), $B(x)$ is also determined for $|x| \leqq 2-2 \varepsilon$. Repeating this procedure, we can determine $b, c$ and $B$ on whole $R$. Their graphs are pictured in Fig. 2. 

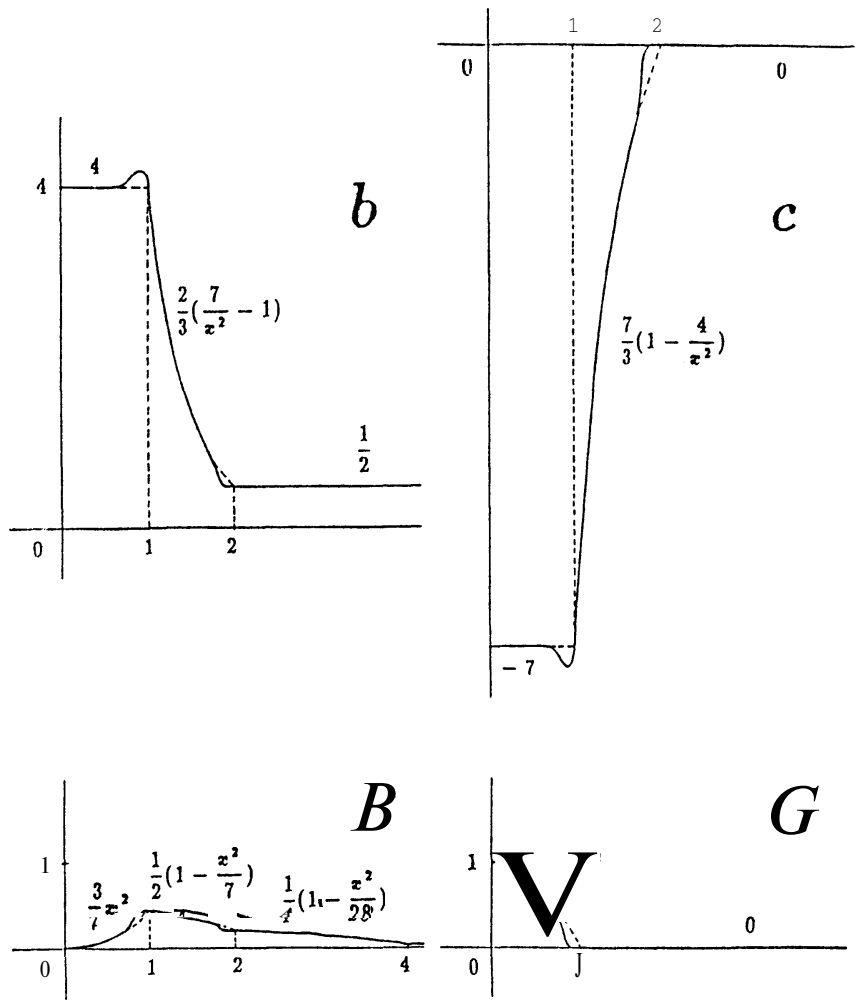

Fig. 2.

Now we define a diffeomorphism $\mathrm{G}$ from a small neighborhood of $A$ onto a small neighborhood of $(1 / 2) A$ by $G(x, y)=(x / 2, b(x) y+\operatorname{sgn}(y) c(x))$,where $\operatorname{sgn}(y)$ -1 if $y>0$ and -1 if $y<0$. Then $G$ is well-defined on a neighborhood of $A$ and $G(A)=(1 / 2) A$. Let $\mathrm{S}$ be the annulus bounded by $A$ and $(1 / 2) A$. By identifying the boundary points of $\mathrm{S}$ by $G$, we obtain from $C V$ a tangentially affine foliation on $T^{2}$ which consists of two Reeb components.

Define a leafwise affine function $F$ on $a$ neighborhood of $\mathrm{S}$ by $F(x, y)=$ $B(x) y+\operatorname{sgn}(y) C(x)$. Then it is easy to check that $F$ satisfies $F=F \circ G$. Hence $F$ induces a leafwise affine function $\bar{F}$ on $T^{2}$. Since $B$ vanishes exactly on the two compact leaves of the foliation, it follows that $\bar{F}$ is nonconstant along each noncompact leaf, as desired.

\section{The space of leafwise affine funcfions}

In this section we investigate the space of all leafwise affine functions for a tangentially affine foliation on $T^{2}$. First we will prove Theorem 2 . 
Proof of Theorem 2. Let $\mathrm{ff}$ and $F$ be as in the assumption of Theorem 2. Denote by $K$ the union of all the compact leaves of $\mathscr{F}$. Let $U$ be a connected component of $T^{2}-K$. The completion $\hat{U}$ of $U$ is either a foliated interval bundle over the circle or a Reeb component. It suffices to show that, in each case, $F \mid \hat{U}$ is uniquely determined by $F \mid d O$.

First we consider the case of a foliated interval bundle. In this case, $\mathscr{F} \mid \hat{U}$ is obtained as follows: Let $g$ be a local diffeomorphism of $R^{2}$ of the form $g(x, y)=(a(x), b(x) y+1)$, where $a(0)=0, a(1)=1$ and $a(x)<x$ for all $0<x<1$ and $b(x)>0$ for all $0 \leqq x \leqq 1$. $\hat{U}$ is obtained from the square $[0,1]^{2}$ by identifying $[0,1] \times\{0\}$ with $[0,1] \times\{1\}$ by $\mathrm{g}$. $\mathscr{F} \mid O$ is induced from the product foliation $x=$ constant. We assume that there exists a nonconstant leafwise affine function $\widetilde{F}$ on $\hat{U} . F$ lifts to a nonconstant function $\mathrm{F}$ on $[0,1]^{2}$ of the form $F(x, y)=$ $B(x) y+C(x)$ satisfying the invariance condition $F \circ g=F$, or equivalently, $B(x)$ $=B(a(x)) b(x)$ and $C(x)=B(a(x))+C(a(x))$. Then we have $B\left(a_{k}(x)\right)=$ $B(x) \prod_{\nu=0}^{k-1} b\left(a_{\nu}(x)\right)^{-1}$ and $C(x)=B(x) \sum_{k=1}^{l} \prod_{\nu=0}^{k-1} b\left(a_{\nu}(x)\right)^{-1}+C\left(a_{l}(x)\right)$, for any fe and $i>0$, where we write $a_{\nu}(x)=a \circ \cdots \circ a(x)$ ( $\nu$ times). Therefore, io $\mathrm{x}$ with $B(x)$ $\neq 0$, the limit $H(x)=\sum_{k=1}^{\infty} \prod_{\nu=0}^{k-1} b\left(a_{\nu}(x)\right)^{\text {-exists and }}$

$$
C(x)=B(x) H(x)+C(0) \text {. }
$$

Also, we have $B\left(a_{-k}(x)\right)=B(x) \prod_{\nu=1}^{k} b\left(a_{-\nu}(x)\right.$ )nd $C(x)=C\left(a_{-l}(x)\right)-B(x) \sum_{k=1}^{l-1} \prod_{\nu=1}^{k}$ $b\left(a_{-\nu}(x)\right)$, for any $k$ and $l>0$, and thus, for $x$ with $B(x) \neq 0$, the limit $K(x)=$ $\sum_{k=0}^{\infty} \prod_{\nu=1}^{k} b\left(a_{-\nu}(x)\right)$ exists and

$$
C(x)=C(1)-B(x) K(x) .
$$

By (1) and (2), we have

and

$$
B(x)=\frac{C(1)-C(0)}{H(x)+K(x)}
$$

$$
C(x)=\frac{C(1) H(x)+C(0) K(x)}{H(x)+K(x)}
$$

for $x$ with $B(x) \neq 0$. Now suppose there is some $0<x<1$ such that $B(x)=0$. Then for all $k \in \boldsymbol{Z}, C(x)=C\left(a_{k}(x)\right)=\mathrm{C}(0)=\mathrm{C}(1)$. This with (3) and (4) implies that $B$ is identically zero and $\mathrm{C}$ is constant, contradicting our assumption. Thus $B(x) \neq 0$ for every $0<x<1$. Hence, by (3) and (4), $F \mid \hat{U}$ is determined by $F \mid \partial \hat{U}$.

Next we consider the case of a Reeb component. In this case, $\mathscr{I} \mid \hat{U}$ is obtained as follows: Let $A$ be the curve consisting of two line segments $\{y-$ $\pm 2,0 \leqq x \leqq 2\}$ and a half circle $\left\{(x-2)^{2}+y^{2}=4, x \geqq 2\right\}$. Put $(1 / 2) A=\{1 / 2(x y) \in$ $\left.\boldsymbol{R}^{2} \mid(x, y) \in A\right\}$. Let $D$ be the closed subset of $\boldsymbol{R}^{2}$ surrounded by two curves $A$ and $(1 / 2) A$ and two line segments $\{x=0,1 \leqq|y| \leqq 2\}$. Let $g$ be a diffeomorphism from a neighborhood of $A$ onto a neighborhood of $(1 / 2) A$ such that $g(x, y)=$ $g+(x, y)$ for $y \geqq 0$ and $g(x, y)=g_{-}(x, y)$ for $y \leqq 0$, where $g_{ \pm}(x, y)=\left(a_{ \pm}(x)\right.$, $\left.b_{ \pm}(x) y+c_{ \pm}(x)\right)$ and $\mathrm{fl}_{ \pm}(0) \mathrm{s}=0, a_{ \pm}(x)<x$ for $x>0, g_{+}=g_{-}$and $c_{ \pm}=0$ near $x=4$. Now, assume that there exists a nonconstant leafwise affine function $\ddot{F}$ on $\hat{U}$. 
$f i$ lifts to a nonconstant function $F$ on $D$ of the form $F(x, y)=F_{+}(x, y)$ for $y \geqq 0$ and $F(x, y)=F_{-}(x, y)$ for $y \leqq 0$, where $F_{ \pm}(x, y)=B_{ \pm}(x) y+C_{ \pm}(x)$, satisfying the condition $F_{+}=F_{-}$near $[2,4] \times\{0\}$ and the invariance condition $F g=F$. Similarly to the foliated bundle case, we see that, for $x$ with $B_{ \pm}(x) \neq 0$, there exists the limit $H_{ \pm}(x)=\sum_{k=1}^{\infty} c_{ \pm}\left(a_{k-1}(x)\right) \prod_{\nu=0}^{k-1} b_{ \pm}\left(a_{\nu}(x)\right)$ āhd it holds that $C_{ \pm}(x)=$ $B_{ \pm}(x) H_{ \pm}(x)+C_{ \pm}(0)$. From this we have

and

$$
B_{ \pm}(x)=\frac{C_{+}(0)-C_{-}(0)}{H_{-}(x)-H_{+}(x)}
$$

$$
C_{ \pm}(x)=\frac{C_{+}(0) H_{-}(x)-C_{-}(0) H_{+}(x)}{H_{-}(x)-H_{+}(x)},
$$

for all $x$ near $[2,4]$. Here $H_{+}(x) \neq H_{-}(x)$, for, otherwise, we must have $C_{+}(0)$ $=C_{-}(0)$. But this implies that $F$ is constant, contradicting the assumption. By a reason similar to the foliated bundle case, $B_{ \pm}(x)$ cannot vanish for any $x>0$. Since it is easy to see that $B_{ \pm}$and $C_{ \pm}$are uniquely determined by their restrictions to a small neighborhood of $[2,4],(5)$ and (6) imply that $\mathscr{F} \mid \hat{U}$ is determined by $\mathscr{I} \mid \partial \hat{U}$. This completes the proof of Theorem 2 .

Let $J L$ be the space of all leaf wise affine functions on $T^{2}$ with respect to $\mathscr{F}$. If $\mathscr{I}$ has no compact leaves, then $\mathcal{A}$ consists only of globally constant functions, hence $\operatorname{dim} A=1$. If $\mathscr{F}$ has compact leaves, then by Theorem 2 we see that $\operatorname{dim} J L$ is not greater than the number of the compact leaves of $\mathscr{F}$. But in order to determine the exact value of $\operatorname{dim} \mathcal{A}$, we need further information on the behavior of leafwise affine functions near compact leaves: Let $g$ be a diffeomorphism of the form $g(x, y)=(a(x), b(x) y+1)$ where $a(0)=0$ and $0<a(x)<x$ and $b(x)>0$ for $0<x \leq 1$. Identifying the side $y=0$ with the side $y-1$ by $g$, we obtain from the product foliation $\mathcal{C V}$ on the rectangle $[0,1]^{2}$ a tangentially affine foliation $\mathscr{F}$ on a cylinder-like surface 5 with corner. $\mathscr{I}$ has a unique compact leaf $L$ corresponding with $x=0$. What we are interested in is the behavior of a leafwise affine function on a small neighborhood of $L$. Let $\mathcal{A}_{0}$ be the vector space of all leafwise affine functions $F$ on $\mathrm{S}$ which vanish on $L$. We may regard $F$ as a function on $[0,1]^{2}$ which is affine in $y$, such that $F(0,30=0$ and $F \circ g=F$. Then we have the following.

THEOREM 3. $\operatorname{dim} \mathcal{A}_{0}$ is 0,1 or $\infty$.

We prove Theorem 3 by a series of lemmas.

Put $q_{k}(x)=\prod_{\nu=0}^{k-1} b\left(a_{\nu}(x)\right)^{-1}$ and $H(x)=\sum_{k=1}^{\infty} q_{k}(x)$.

Let $B(x) y+C(x) \in \mathcal{A}_{0}$. Then $B$ and $C$ satisfy the invariance condition $B(x)$ $=B(a(x)) b(x)$ and $C(x)=B(a(x))+C(a(x))$. By this condition, we have $B\left(a_{k}(x)\right)$ $=B(x) q_{k}(x), \quad C(x)=B(x) H(x)$ and $\quad C\left(a_{n}(x)\right)=B(x) \sum_{k=n+1}^{\infty} q_{k}(x)$ for $\quad 0<x \leqq 1$ and fe, rc $>0$.

Recall that a function $\varphi$ is said to be flat at 0 if $\varphi^{(r)}(0)=0$ for all $r \in N$.

LEMMA 1. // there exists $F(x, y)=B(x) y+C(x) \in \mathcal{A}_{0}$ such that $B$ or $C$ is 
nonflat at 0 , then $\operatorname{dim} \mathcal{A}_{0}=1$.

Proof. Since $B(x)=C\left(a_{-1}(x)\right)-C(x)$ if $\mathrm{C}$ is flat, so is $B$. So we may assume that $C$ is nonflat. Suppose there exists another $B_{1}(x) y+C_{1}(x) \in \mathcal{A}_{0}$ which is not a scalar multiple of $F$. For $x>0$ near 0 , put $E(x)=B_{1}(x) / B(x)\left(=C_{1}(x) /\right.$ $C(x)$ ). Then $E$ is not a constant function and satisfies that $E(x)=E(a(x))$. From this and the nonflatness of $\mathrm{C}$ it follows easily that $E(x) C(x)$ cannot extend to a smooth function at 0 , which contradicts that $C_{1}(x)=E(x) C(x)$.

LEMMA 2. $/ / a^{\prime}(0)<1$, then $\operatorname{dim} \mathcal{A}_{0} \leqq 1$.

Proof. Choose constants $0<\alpha<1, \quad \beta>0$ and $m \in N$ so that $b(x)<\beta$ and $a(x)$ $<\alpha x$ for $0<x \leqq 1$, and that $\alpha^{m} \beta<1$. Let $\mathrm{F}$ be any element of $A_{0}$ and $B$ the coefficient of $y$ in F. Suppose that $B(x) \neq 0$ for some $x>0$. Since $B\left(a_{n}(x)\right)=$ $B(x) / \prod_{k=0}^{n-1} b\left(a_{k}(x)\right)$, we have

$$
\frac{B\left(a_{n}(x)\right)}{\left(a_{n}(x)\right)^{m}}=\frac{B(x)}{\left(a_{n}(x)\right)^{m} \Pi_{k=0}^{n-1} b\left(a_{k}(x)\right)} \geqq \frac{B(x)}{\left(\alpha^{n} x\right)^{m} \beta^{n}}-\frac{B(x)}{x^{m}} \frac{1}{\left(\alpha^{m} \beta\right)^{n}} \longrightarrow \infty
$$

as $n$ tends to $\infty$. This implies that $B$ is not flat at 0 . Then by Lemma 1, we have that $\operatorname{dim} A_{0} \leqq 1$. This proves Lemma 2 .

We define $\bar{B}$ by $\bar{B}(x)=\prod_{\nu=0}^{\infty} b\left(a_{\nu}(x)\right) / b\left(a_{\nu}(1)\right)$. The following lemmas are proved by some calculations.

LEMMA 3. $\bar{B}(x)$ is $C^{\infty}$ and positıve for $0<x \leqq 1$, and satisfies $\bar{B}(x)=$ $\bar{B}(a(x)) b(x) / b(0)$.

LEMMA 4. // $H\left(x_{0}\right)<\infty$ for some $x_{0}>0$, then $H(x)$ is $C^{\infty}$ and positive for $0<x \leqq 1$, and $H\left(a_{n}(x)\right) / H\left(a_{n}\left(x_{0}\right)\right.$ converges to $1 C^{\infty}$ '-uniformlyon any compact subset of $(0,1]$.

Recall that there exists a $C^{1}$ function / (which is $C^{\infty}$ for $x>0$ ) such that $a$ is the time one map of the vector field $-f(x) \partial / \partial x$ (See [Se]).

The following lemma is easily proved by induction.

LEMMA 5. Let $E$ and $C$ be $C^{\infty}$ functions on $(0,1]$ such that $E(a(x))=E(x)$ for all $0<x \leqq 1$. Then, for each $l \geqq 0$ and $x$ with $C\left(a_{n}(x)\right) \neq 0$, we have

$$
(E C)^{(l)}\left(a_{n}(x)\right)=C\left(a_{n}(x)\right) f\left(a_{n}(x)\right)^{-l} F_{l}
$$

where $g_{n}(x)=\left(\log \left|C\left(a_{n}(x)\right)\right|\right)^{\prime}$ and $F_{l}$ is a polynomial in the functions $E^{(i)}(x)$, $f^{(i)}(x), g_{n}^{(i)}(x)$ and $f^{(i)}\left(a_{n}(x)\right) f\left(a_{n}(x)\right)^{2-\gamma(0 \leqq i \leqq l)}$ which is defined inductively as follows $\cdot F_{0}=E, F_{l+1}=\left(f g_{n}+f D_{x}\right) F_{l}+\left(-l f^{\prime}\left(a_{n}\right)+f\left(a_{n}\right) D_{a_{n}}\right) F_{l}$.

We make the following remark: Assume that $H$ satisfies the hypothesis of Lemma 4. Let $B$ and $\mathrm{C}$ be $C^{\infty}$ functions on $(0,1]$ such that $B(x)=B(a(x)) b(x)$ and $\quad C(x)=B(x) H(x)$. Then $g_{n}(x)=\left(\log \mid B(x) q_{n}(x) H\left(a_{n}(x)\right) / q_{n}(1) H\left(a_{n}(1)\right) \phi \varnothing n-\right.$ 
verges to $g(x)=(\log |B(x) / \bar{B}(x)|)^{\prime}$ for $x$ with $B(x) \neq 0$. If we further assume $\mathrm{fl}^{\prime}(0)=1$, then since $\lim _{x \rightarrow 0} f^{(i)}(x) f(x)^{2-1}=0$ ([Se, 3.6]), $F_{l}(x)$ converges to some function $G_{l}(x)$ as $n \rightarrow \infty$ for $x$ with $B(x) \neq 0$. And we have $G_{l+1} / f=g G_{l} G_{l}^{\prime}$.

LEMMA 6. $/ / a^{\prime}(0)=1$ and if for any $l \geqq 0$ there exists $x_{0}>0$ such that $f\left(a_{n}\left(x_{0}\right)\right)^{-1} \sum_{k=n+1}^{\infty} q_{k}\left(x_{0}\right)$ converges to 0 as $n \rightarrow \infty$, then $\operatorname{dim} \mathcal{A}_{0}=\infty$.

Proof. Put $\Delta x=x-a(x)$. By [Se, 2.9] and Lemma 4, we have

$$
\frac{f\left(a_{n}(x)\right)^{-l} \sum_{k=n+1}^{\infty} q_{k}(x)}{f\left(a_{n}\left(x_{0}\right)\right)^{-l} \sum_{k=n+1}^{\infty} q_{k}\left(x_{0}\right)} \sim\left(\frac{\Delta a_{n}(x)}{\Delta a_{n}\left(x_{0}\right)}\right)^{-l} \frac{q_{n}(x) H\left(a_{n}(x)\right)}{q_{n}\left(x_{0}\right) H\left(a_{n}\left(x_{0}\right)\right)} \rightarrow \frac{\bar{B}\left(x_{0}\right)}{\bar{B}(x)}
$$

as $n \rightarrow \infty$. Hence, by the hypthesis, we have that $f\left(a_{n}(x)\right)^{-l} \sum_{k=n+1}^{\infty} q_{k}(x)$ converges to 0 uniformly on any compact subset of $(0,1]$. Now, take arbitrary $C^{\infty}$ functions $B_{1}>0$ and $B_{0}$ on $[a(1), 1]$ satisfying $\left(B_{i}(a) b\right)^{(r)}(1)=B_{i}^{(r)}(1)$ for each $r \geqq 0$ and $i=0,1$. We define $C^{\infty}$ functions $\tilde{B}_{\imath}$ and $\tilde{C}_{\imath}$ on $(0,1]$ by $\widetilde{B}_{i}\left(a_{k}(x)\right)=$ $B_{i}(x) q_{k}(x)$ and $C_{i}(x)=\tilde{B_{i}}(x) H(x)$. Applying Lemma 5 to $C=C_{1}$ and $E=C_{0} / C_{1}$, we have, for any $l \geqq 0$,

$$
\tilde{C}_{0}^{(l)}\left(a_{n}\right)=\tilde{B}_{1} f\left(a_{n}\right)^{-l} \sum_{\mathrm{fe}=\mathrm{n}+1}^{n} q_{k} F_{l} \longrightarrow 0
$$

as $n \rightarrow \infty$ uniformly on $[a(1), 1]$. Thus $\tilde{C_{0}}$ (and hence also $\widetilde{B}_{0}$ ) is extended to a $C^{\infty}$ function on $[0,1]$ (and is flat at 0 ). Therefore $\widetilde{B}_{0}(x) y+\widetilde{C}_{0}(x) \in \mathcal{A}_{0}$. Hence $\operatorname{dim} \mathcal{A}_{0}=\infty$, completing the proof.

LEMMA 7. // $\mathrm{fl}^{\prime}(0)=1$ and if there exists $l_{0} \geqq 0$ such that, for any $x>0$, $f\left(a_{n}(x)\right)^{-l_{0}} \sum_{k=n+1}^{\infty} q_{k}(x)$ does not converge to 0 as $n-\rightarrow \infty$. Then $\operatorname{dim} \mathcal{A}_{0}$ is either 0 or 1 .

Proof. Assume that $\mathcal{A}_{0}$ contains a nonzero function $B(x) y+C(x)$. Put $l=l_{0}+1$. Then, applying Lemma 5 with $E=l$, we have

$$
C^{(l)}\left(a_{n}(x)\right)=B(x) f\left(a_{n}(x)\right)^{-l} \sum_{\mathbf{f t}=\mathbf{n}+\mathbf{l}}^{\infty} q_{k}(x) F_{l}
$$

Since this quantity must converge as $n \rightarrow \infty$, we have $G_{l}(x)=\lim _{n \rightarrow \infty} F_{l}(x)=0$ for $x$ with $B(x) \neq 0$. If we solve the first order linear differential equations $G_{m}^{\prime}+$ $g G_{m}=G_{m+1} / f(0 \leqq m<l)$ successively (downward on $m$ ), we finally obtain the equality $G_{0}(x) \exp \int^{x} g(x) d x=\lambda B(x) / \bar{B}(x)=\sum_{i=0}^{l-1} \lambda_{i} h(x)$ on each connected component of the set $\{x \mid B(x) \neq 0\}$, where $\lambda \neq 0$ and $\lambda_{2}$ are constants and $h(x)=$ $\int_{1}^{x} d x / f(x)$. We may consider $h$ as a coordinate on $(0,1]$. Since $(d / d h)^{l}(B / B)$ $\Xi \Xi O, B \bar{T} B$ is a polynomial in $h$ on $(0,1]$. If we replace $x$ by $a(x)$, then $B / \bar{B}$ is multiplied by $1 / b(0)$ while $h(a(x))=h(x)-1$. From this, we have that $\lambda_{2}=0(i \geqq 1)$ and we can conclude that $B$ must be a constant multiple of $\bar{B}$. Lemma 7 is proved. 
The proof of Theorem 3 is complete.

Remark. By making a little more effort, we can describe the structure of $\mathcal{A}_{0}$ concretely: In the case where $\operatorname{dim} A_{0}=\infty$,

$$
\mathcal{A}_{0}=\left\{\widetilde{B}_{0}(x)\left(y+H(x) \mathrm{I} B_{0} \text { is as in the proof of Lemma } 6\right\} .\right.
$$

In the case where $\operatorname{dim} \mathcal{A}_{0}=1$, either of the following holds: (i) $a^{\prime}(0)<1$ and $b(0)=a^{\prime}(0)^{-m}(m \in N)$, or (ii) $\beta^{\prime}(0)=1$ and $\mathrm{ft}(0)=1$. Ih the case (i), by [St], there is a diffeomorphism $\phi$ such that $\phi\left(a\left(\phi^{-1}(x)\right)\right)=a^{\prime}(0) x$. Define $B$ by $\hat{B}(x)=$ $\phi(x)^{m} \bar{B}(x)$. Then

$$
\mathcal{A}_{0}=\{c \hat{B}(x)(y+H(x)) \mid c \in \boldsymbol{R}\} .
$$

In the case (ii),

$$
\mathcal{A}_{0}=\{c \bar{B}(x)(y+H(x)) \mathrm{I} c \in \boldsymbol{R}\} .
$$

In various cases we can calculate the dimension of $\mathcal{A}_{0}$

\section{Example 3.}

Case I. $a^{\prime}(0)<1$.

(i) $b(0)=a^{\prime} \mid(0)^{-m}$ (for some $m \in N$ ) $\Rightarrow \operatorname{dim} A_{0}=1$.

(ii) otherwise $\Rightarrow \operatorname{dim} A_{0}=0$.

Case II. $\quad a(x)=x-x^{n}+\cdots(n \geqq 2)$.

(i) $b(0)>1 \Rightarrow \operatorname{dim} A_{0}=\infty$.

(ii) $b(0)<1 \Rightarrow \operatorname{dim} \mathcal{A}_{0}=0$.

(iii) $b(x)=1+\beta x^{l}+\cdots(1 \leqq l \leqq n-2, \beta>0) \Rightarrow \operatorname{dim} \mathcal{A}_{0}=\infty$.

(iv) $b(x)=1+\beta x^{l}+-(1 \leqq l \leqq n-2, \beta<0) \Rightarrow \operatorname{dim} \mathcal{A}_{0}=0$.

(v) $b(x)=1+m x^{n-1}+\cdots(\boldsymbol{Z} \ni m \geqq n) \Rightarrow \operatorname{dim} \mathcal{A}_{0}=1$.

(vi) other wise $\Rightarrow \operatorname{dim} \mathcal{A}_{0}=0$.

Case III. $a(x)=x-$ (a positive flat function).

(i) $b(0)>1 \Rightarrow \operatorname{dim} A_{0}=\infty$.

(ii) $b(0)<1 \Rightarrow \operatorname{dim} A_{0}=0$.

(iii) $b(x)=1+\beta x^{l}+\cdots(1 \leqq l, \beta>0) \Rightarrow \operatorname{dim} A_{0}=\infty$.

(iv) $b(x)=1+\beta x^{l}+\cdots(1 \leqq l, \beta<0) \Rightarrow \operatorname{dim} A_{0}=0$.

(v) $b(x)=1+($ a flat function).

(v-1) $\forall l \exists x$ such that $f\left(a_{n}(x)\right)^{-1} \sum_{k=n+1}^{\infty} q_{k}(x) \rightarrow 0 \Rightarrow \operatorname{dim} A_{0}=\infty$.

(v-2) not $(\mathrm{v}-1)$, but $\bar{B}$ and $\bar{B} H$ are $C^{\infty}$ on $[0,1] \Rightarrow \operatorname{dim} A_{0}=1$.

(v-3) otherwise $\Rightarrow \operatorname{dim} A_{0}=0$.

If we use Theorems 2, 3 and Example 3, we can determine the space of all leafwise affine functions on a tangentially affine foliations on $T^{2}$ in almost all cases.

\section{A remark on the 3-dimensional case}

Let $\mathscr{I}$ be a codimension one foliation on a closed 3-manifold. Then an 
obvious necessary condition for $\mathscr{F}$ to admit a tangentially affine structure is that each leaf can admit at least one affine manifold structure. Proposition shows, however, that the condition is not sufficient.

Proof of Proposition. Let $L$ be the torus leaf of the Reeb foliation $\mathscr{F}$. Choose two oriented loops $a$ and $\beta$ in $L$ so that $a$ is a vanishing cycle in the one Reeb component and that $\beta$ is a vanishing cycle in the other Reeb component. Denote by $\Phi: \pi_{1}(L) \rightarrow \operatorname{Aff}(\boldsymbol{R}$ the holonomy homomorphism (for definition, see $[\mathrm{GH}]$ ) associated with the affine structure of $L$. Let $\left\{\alpha_{t}\right\}_{0 \leq t<1}$ be a continuous family of loops such that $\alpha_{0}=\alpha$ and that $\alpha_{t}, t>0$, lies in a plane leaf $L_{t}$. Then, obviously, for any $t>0$ the holonomy along $\alpha_{t}$ induced from the affine structure of $L_{t}$ is the identity because of the 1-connectedness of $L_{t}$. This with the fact that $\alpha_{t}$ converges to $a$ implies that the holonomy $\Phi(\alpha)$ must be the identity. By the same reason, $\Phi(\beta)$ is also the identity. Consequently $\Phi$ must be trivial, which is absurd. This proves the proposition.

\section{REFERENCES}

[AN] ARnol'D, V.I. AND NOVIKOV, S.P. (eds), Dynamical Systems IV. Encyclopaedia of Math. Sci., Vol. 4. Berlin, Heidelberg, New York: Springer 1990.

[BG] BOYER, C. P. AND GITLER, S., The theory of $G^{\infty}$-supermanifolds. Trans. Amer. Math. Soc. 285, 241-267 (1984).

[CRT] CATENACCI, R., REINA, C. AND TE'OFILATTO, P., On the body of supermanifolds. J. Math. Phys. 26, 671-674 (1985).

[Fr] FRIED, D., Polynomials on affine manifolds. Trans. Amer. Math. Soc. 274, 709719 (1982).

[Fu] FURNESS, P., Affine foliations of codimension one. Quart. J. Math. 25, 151-161 (1974).

[GH] GOLDMAN, W. M. AND HIRSCH, M.W., Polynomial forms on affine manifolds. Pacific J. Math. 101, 115-121 (1982).

[In] INABA, T., Resilient leaves in transversely affine foliations. Tסhoku Math. J. 41, 625-631 (1989).

[RC1] RABIN, J.M. AND CRANE, L., Global properties of supermanifolds. Commun. Math. Phys. 100, 141-160 (1985).

[RC2] RABIN, J.M. AND CRANE, L., How different are the supermanifolds of Rogers and DeWitt? Commun. Math. Phys. 102, 123-137 (1985).

[Ro] ROGERS, A., A global theory of supermanifolds. J. Math. Phys. 21, 1352-1365 (1980).

[Se] SERGERAERT, F., Feuilletages et difféomorphismesinfiniment tangents $a$ l'identité. Invent. Math. 39, 253-275 (1977).

[St] Sternberg, S., Local $C^{n}$ transformations of the real line. Duke Math. J. 24, 97102 (1957).

[Ts] TSUCHIYA, N., On elementary transversely affine foliations I. Kodai Math. J. 13, 289-297 (1990). 
COLLEGE OF ARTS AND SCIENCES,

Chiba UNIVERSITY, Chiba 260,

JAPAN.

DEPARTMENT OF MATHEMATICS,

TOKYO InsTiTUTE OF TECHNOLOGY,

OH-OKAYAMA, TOKYO 152,

JAPAN. 